\title{
Potential risks of microplastics combined with superbugs: Enrichment of antibiotic resistant bacteria on the surface of microplastics in mariculture
} system

\author{
Yuxuan Zhang ${ }^{\mathrm{a}, \mathrm{b}}$, Jian $\mathrm{Lu}^{\mathrm{a}, \mathrm{b}, \mathrm{c}, *}$, Jun $\mathrm{Wu}^{\mathrm{d}}$, Jianhua Wang ${ }^{\mathrm{a}}$, Yongming Luo ${ }^{\mathrm{a}}$ \\ ${ }^{a}$ CAS Key Laboratory of Coastal Environmental Processes and Ecological Remediation, Yantai Institute of Coastal Zone Research (YIC), Chinese Academy of Sciences \\ (CAS), Shandong Key Laboratory of Coastal Environmental Processes, YICCAS, Yantai, Shandong, 264003, PR China \\ ${ }^{\mathrm{b}}$ University of Chinese Academy of Sciences, Beijing, 100049, PR China \\ ${ }^{\mathrm{c}}$ Center for Ocean Mega-Science, Chinese Academy of Sciences, 7 Nanhai Road, Qingdao, 266071, PR China \\ ${ }^{\mathrm{d}}$ School of Resources and Environmental Engineering, Ludong University, Yantai, Shandong, 264025, PR China
}

\section{A R T I C L E I N F O}

\section{Keywords:}

Microplastic

Antibiotic resistant bacteria

Superbugs

Multi-drug resistance

Antibiotic resistance genes

Mariculture

\begin{abstract}
A B S T R A C T
Microplastics have become emerging pollutants and served as potential vectors for harmful bacteria, while rare information on the emergency and propagation of antibiotic resistant bacteria (ARB) and antibiotic resistance genes (ARGs) on the surface of microplastics is available. This study investigated the enrichment of ARB, especially multi-antibiotic resistant bacteria (MARB), on the surface of microplastics in mariculture system. Polyethylene terephthalate accounted for the highest proportion (75\%) in the collected microplastics. The counts of cultivable ARB in microplastic samples were $6.40 \times 10^{6}-2.48 \times 10^{8} \mathrm{cfu} / \mathrm{g}$, which were $100-5000$ times higher than those in water samples. The ratios of cultivable ARB to total cultivable bacteria from microplastic samples were higher than those from water samples. High-throughput sequencing showed that the diversity and abundance of cultivable ARB in the microplastic samples was high with the predominant bacterial genera of Vibrio, Muricauda and Ruegeria. Total 160 MARB isolates were obtained and most of isolates were obtained from the microplastic samples. MARB isolates resisting or intermediating to four and three antibiotics accounted for much higher proportions in the microplastic samples, and the higher percentage of antibiotic resistance was to penicillin, sulfafurazole, erythromycin and tetracycline. The dominant multiple antibiotic resistance profile was TET-SFX-ERY-PEN, which accounted for $25.4 \%$ in microplastic samples and $23.9 \%$ in water samples. In typical MARB isolates, the positive detection rate of ARGs was up to $80.0 \%$ in microplastic samples while that was $65.3 \%$ in water samples. Five types of class 1 integrons (intI1) associated gene cassette arrays and seven types of gene cassettes were detected in microplastic samples, which were more than those in water samples. These results revealed that microplastics were hazardous pollutants for the enrichment of ARB, especially superbugs, and the spread of antibiotic resistance.
\end{abstract}

\section{Introduction}

As emerging marine pollutants, microplastics ( $<5 \mathrm{~mm}$, MP) have drawn considerable attention (Law and Thompson, 2014; Thompson et al., 2004) since they have been found in water and sediments as well as global marine ecosystems such as oceans, lakes, rivers and polar water (Lusher et al., 2015). It has been reported that the abundance of microplastics reached up to 8654 items per $\mathrm{m}^{3}$ on the sea surface (Hidalgo et al., 2012). Microplastics can absorb pollutants, such as heavy metals, persistent organic pollutants and high toxic emerging pollutants on their surface (Hirai et al., 2011; Lechner et al., 2014). Due to the hydrophobic surface of the polymer in seawater, microplastics can provide attachment points and relatively stable habitats for the microorganisms (Reisser et al., 2014), promote the enrichment of microorganisms, accelerate the formation of biofilms (Lobelle and Cunliffe, 2011), and become the vector for harmful microorganisms. Harmful microorganisms will be transferred to other ecosystems with the transport of microplastics by the water flow, causing changes of microbial communities and the spread of harmful microbes, even causing the spread of infections. A variety of microbial communities

\footnotetext{
* Corresponding author. CAS Key Laboratory of Coastal Environmental Processes and Ecological Remediation, Yantai Institute of Coastal Zone Research (YIC), Chinese Academy of Sciences (CAS), Shandong Key Laboratory of Coastal Environmental Processes, YICCAS, Yantai, Shandong, 264003, PR China.

E-mail address: jlu@yic.ac.cn (J. Lu).
} 
were found to be enriched on the surface of polypropylene (PP) and polyethylene (PE) plastic fragments, with higher quantity than those in surrounding aquatic environment (Zettler et al., 2013). Vibrio and Colwell attached to low density polyethylene, formed biofilms through microbial aggregation and contributed to the degradation of microplastics (Harrison et al., 2014). Microplastics are easily eaten by marine organisms, which cannot distinguish preys and microplastics (Syberg et al., 2015; Wright et al., 2013), and subsequently cause physical (Browne et al., 2013), chemical (Rochman, 2013, 2015) and biological (Kiessling et al., 2015; Zettler et al., 2013) hazard to ecological environment (Frias et al., 2010) and marine organisms in the food chain.

In industrial recirculating maricultural systems, microplastics dropped from the biofilter materials could be continuously accumulated with the circulation of aquaculture water, colonized by microorganisms and wrapped by biofilms. As drugs for the prevention and treatment of microbial diseases, antibiotics have been widely used as growth promoters in aquaculture (Lu et al., 2018; Wang et al., 2019). However, antibiotics have been abused in mariculture systems due to the lacking of scientific management and policy. Tetracycline, sulfonamide, quinolone, macrolide, chloramphenicol, $\beta$-lactamase and aminoglycoside are the common classes of antibiotics used in mariculture. Microplastics have been found to influence the evolution of bacterial communities and increase the exchange of genes. Antibiotics attached on the microplastics may induce the emergence and enrichment of antibiotic resistant bacteria (ARB), multi-antibiotic resistant bacteria (MARB) and superbugs, promote the spread of antibiotic resistance genes (ARGs) (Lu et al., 2019; Wang et al., 2018), and the effective dose of antibiotics to kill bacteria keep increasing (Pruden et al., 2006; Zhu et al., 2013). The microplastics are proposed to be vector and hotspot for ARB, ARGs and horizontal gene transfer (HGT). The increasing and spreading of antibiotic resistant pathogenic bacteria, which were hard to be killed, could cause toxicity and threat to organisms and humans, and potential worldwide risks to ecological environment with the transfer and widespread of microplastics.

It is regretful that investigations about the enrichment and proliferation of ARB on the surface of microplastics are not available. Therefore, the differences between cultivable ARB on the surface of microplastics and in surrounding water were investigated by comparing the cultivable ARB community structure, MARB isolates, antibiotic resistance, ARG profiles and intI 1 associated gene cassettes. The objective of present study was to reveal the enrichment of cultivable ARB on the surface of microplastics, which was distinctive from those in water columns in industrial mariculture system. This investigation provided initial information on the enrichment of ARB, especially the MARB (namely superbugs), on the surface of microplastics, and the potential risks of microplastics to ecological environment and human health. To the best of our knowledge, this is the first study on investigating the coselection of multiple antibiotic resistance in bacterial pathogens colonies on microplastics, and it will improve the understanding of the potential risks of microplastics.

\section{Materials and methods}

\subsection{Sampling}

Sampling has been done in an industrial mariculture system with recirculation unit in Yantai City. A total of 3 water samples and 3 microplastic samples were collected from the fish pond (PW, PM), the inlet (IW, IM) and outlet (OW, OM) of fish pond, respectively. Samples were collected in triplicate (subsample-1, subsample-2 and subsample-3) from each sampling point. Water samples were collected with clean and sterile bottles, then filtered through $0.22 \mu \mathrm{m}$ mixed cellulose esters membranes. Microplastics were collected using nets with $300 \mu \mathrm{m}$ mesh in the influent, effluent and pond water. The microplastics separated from the net were filtered by membranes with pore sizes of $0.22 \mu \mathrm{m}$. Water samples were filtered through $0.45 \mu \mathrm{m}$ mixed cellulose esters membranes for analysis of total nitrogen (TN), total phosphorus (TP), ammonia, nitrates, nitrites, phosphates and total organic carbon (TOC). All samples were stored in iceboxes and transported immediately to laboratory. All membranes were stored at $-80^{\circ} \mathrm{C}$ for further analysis.

\subsection{Physicochemical analysis and morphological characterization}

Temperature, dissolved oxygen (DO) and turbidity were measured in situ using a DO monitor (JPB-607A, INESA, China) and a portable multi-parameter water quality measuring instrument (5B-2H (V8), Lian-hua Tech, China). Salinity was measured by a portable refractometer (LH-Y100, Lohand Biological, China), and $\mathrm{pH}$ was determined by a $\mathrm{pH}$ meter (PHS-3C, INESA, China). Ammonia, nitrates, nitrites, phosphates, TN and TP were analyzed by a continuous flow analyzer (Auto Analyzer III, Seal, Germany). TOC was measured with a total organic carbon analyzer (TOC-VCPH, Shimadu, Japan). The basic water quality parameters were shown in Table S1.

The physical parameters of microplastics on the surface of membranes after filtration were characterized by stereomicroscope (Lerca S9i, Germany). Characterization and functional groups of microplastics were identified by Fourier Transform Infrared Spectroscopy (FTIR, Nicolet iS5, Thermo Fisher, USA). The FTIR spectra were collected in the spectral range between 4000 and $650 \mathrm{~cm}^{-1}$ with repeating 32 times, and then compared with the HR Nicolet Sampler Library.

\subsection{High-throughput sequencing of cultivable $A R B$}

The investigation on the abundance and diversity of all ARB is still a challenge. The investigation of antibiotic resistant bacteria (ARB) is currently based on the investigation of cultivable ARB, which is rational and traditional. Therefore, the abundance and diversity of ARB were evaluated based on the investigation of cultivable ARB in present work. In order to investigate the abundance and diversity of the cultivable $\mathrm{ARB}$ on the surface of microplastics in recirculating mariculture system, Zobell 2216E agar plates were used to cultivate ARB. All agar plates were divided into three kinds: blank plates without antibiotics, antibiotic selective plates with one antibiotic, and plates with a combination of seven antibiotics. One gram of microplastics or $1 \mathrm{~mL}$ water sample was homogenized in $10 \mathrm{~mL}$ sterilized seawater. The homogenized samples were diluted and serial 10 -fold dilutions up to $10^{8}$ prepared with autoclaved seawater. A volume of $100 \mu \mathrm{L}$ diluent was plated onto three Zobell $2216 \mathrm{E}$ agars and then incubated at $30^{\circ} \mathrm{C}$ for 24-48 h. Antibiotics were classified into seven classes: tetracycline (tetracycline), sulfonamide (sulfafurazole), quinolone (ciprofloxacin), chloramphenicol (chloramphenicol), $\beta$-lactamase (penicillin), aminoglycoside (gentamicin) and macrolide (erythromycin). Antibiotic stock solutions were prepared and diluted to working concentrations for use in susceptibility test following Clinical and Laboratory Standards Institute (CLSI) guidelines (Wayne, 2011). Working concentrations were higher than the minimum inhibitory concentrations (MIC) for resistant bacteria. Plates without antibiotics were used to detect total cultivable bacteria (TCB), plates with single antibiotic were used to obtain ARB, and plates with seven classes of antibiotics were used to cultivate multi-antibiotic resistant bacteria (MARB). For determining the diversity of ARB, bacteria from different types of plates were harvested and the total genomic DNA was extracted for sequencing.

Genomic DNA was extracted by the E.Z.N.A. ${ }^{\circledR}$ soil DNA Kit (Omega Bio-tek, Norcross, GA, U.S.). DNA concentrations, purification and quality were determined by NanoDrop (2000) (Thermo Scientific, Wilmington, USA) and 1\% agarose gel electrophoresis. Purified DNA was sent to Majorbio (Shanghai, China) for analysis. The V4-V5 regions of the bacteria 16S rRNA genes were amplified with primers $515 \mathrm{~F}$ ( 5'-GTGCCAGCMGCCGCGG-3') and 907R (5'-CCGTCAATTCMTTTRAGTTT-3') by PCR system (GeneAmp 9700, ABI, USA). PCR reactions were conducted using following program: $3 \mathrm{~min}$ for denaturation at $95^{\circ} \mathrm{C}, 27$ cycles of $30 \mathrm{~s}$ at $95^{\circ} \mathrm{C}, 30 \mathrm{~s}$ for annealing at $55^{\circ} \mathrm{C}$, and $45 \mathrm{~s}$ for 
elongation at $72{ }^{\circ} \mathrm{C}$, and a final extension at $72{ }^{\circ} \mathrm{C}$ for $10 \mathrm{~min}$. PCR reactions were performed with $20 \mu \mathrm{L}$ mixture containing $5 \times$ FastPfu Buffer $(4 \mu \mathrm{L}), 2.5 \mathrm{mM}$ dNTPs $(2 \mu \mathrm{L})$, forward and reverse primers $(5 \mu \mathrm{M}$, $0.8 \mu \mathrm{L})$, FastPfu Polymerase $(0.4 \mu \mathrm{L})$ and template DNA (10 ng). Products of PCR amplification were examined by $2 \%$ agarose gels and purified by AxyPrep DNA Gel Extraction Kit (Axygen Biosciences, Union City, CA, USA). Purified amplicons were pooled in equimolar and paired-end sequenced $(2 \times 300)$ on an Illumina MiSeq platform (Illumina, San Diego, USA) according to the standard protocols by Majorbio Bio-Pharm Technology Co. Ltd. (Shanghai, China). Raw reads were deposited into NCBI database (Accession Number: SRP212853).

\subsection{Isolation and identification of MARB}

Plates with a combination of seven antibiotics were used to cultivate and isolate MARB. Based on morphology, size and color, MARB isolates were selected from agar plates of six different samples. Genomic DNA was extracted from MARB isolates by TIANamp Bacteria DNA Kit (Tiangen Biotech Co., LTD, China) and used as template DNA to amplify the 16S rRNA genes with primer pair 27F (5'-AGAGTTTGATCCTG GCTCAG-3') and 1492R (5'-GGCTACCTTGTTACGACTT-3'). PCR condition was $3 \mathrm{~min}$ for denaturation at $95^{\circ} \mathrm{C}, 30$ cycles of $30 \mathrm{~s}$ at $95^{\circ} \mathrm{C}$, $30 \mathrm{~s}$ for annealing at $55^{\circ} \mathrm{C}$, and $60 \mathrm{~s}$ for elongation at $72{ }^{\circ} \mathrm{C}$, and a final extension at $72^{\circ} \mathrm{C}$ for $10 \mathrm{~min}$. PCR products were purified and sent to the Beijing Genomics Institute (BGI) for sequencing. All sequence alignments were conducted by a blast search in NCBI database.

\subsection{Antibiotic susceptibility testing}

The MARB isolates were tested for their susceptibility to antibiotics by Kirby-Bauer (KB) disc diffusion on Müller-Hinton (MH) agar plates. Seven types of KB discs were selected for testing: tetracycline $(30 \mu \mathrm{g})$, sulfafurazole $(300 \mu \mathrm{g})$, ciprofloxacin $(5 \mu \mathrm{g})$, chloramphenicol $(30 \mu \mathrm{g})$, penicillin $(10 \mu \mathrm{g})$, gentamicin $(10 \mu \mathrm{g})$ and erythromycin $(15 \mu \mathrm{g})$ (Hangzhou Microbial Reagent Co., LTD, China). Antimicrobial susceptibility tests and selection of antibiotic discs were conducted according to Clinical and Laboratory Standards Institute (CLSI) guidelines (Wayne, 2011). Strain of Escherichia coli ATCC 25922 was used as negative control. The inoculum of each isolate was suspended in sterile saline solution $(0.85 \% \mathrm{NaCl})$ to match $0.5 \mathrm{McFarland}$ standards in turbidity and streaked on $\mathrm{MH}$ agar with a swab. Plates were incubated at $37^{\circ} \mathrm{C}$ for $24 \mathrm{~h}$ and the inhibition zone diameters were measured to determine the antibiotic susceptibility of all the MARB isolates.

\subsection{Detection of ARGs and MGEs in MARB}

A total of 75 ARGs (94 assays) and class 1 integrons (intI 1 and intI1V) were examined in the typical MARB isolates. These selected ARGs belonged to eight classes, including tetracycline (tet $\mathrm{A}$, tet $\mathrm{B}$, tet $\mathrm{C}$, tet $\mathrm{D}$, tet $\mathrm{E}$, tet $\mathrm{G}$, tet $\mathrm{K}$, tet $\mathrm{L}$, tet $\mathrm{M}$, tet $\mathrm{O}$, tet $\mathrm{Q}$, tet $\mathrm{S}$, tet $\mathrm{T}$, tet $\mathrm{W}$, tet $\mathrm{X}$ ), sulfonamide (sul1, sul2, sul3), quinolone (qnrA, qnrB, qnrS), chloramphenicol

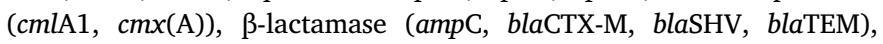
aminoglycoside (aac(6')-Ib, aacA/aphD, aac 2 , aacC4, aadA1, aphA3, str), macrolide (ereA, ereB, mphA), and multidrug. Genomic DNA was extracted from the MARB isolates and used as templates for PCR amplification. Primers for PCR amplification were listed in Table S2. Each $50 \mu \mathrm{L}$ PCR mixture contained $1.0 \mu \mathrm{L}$ forward primer $(20 \mu \mathrm{M}), 1.0 \mu \mathrm{L}$ reverse primer $(20 \mu \mathrm{M}), 1.0 \mu \mathrm{L}$ template DNA, $0.25 \mu \mathrm{L}$ TaKaRa Taq $(5 \mathrm{U} /$ $\mu \mathrm{L}$ ), $5.0 \mu \mathrm{L} 10 \times$ PCR Buffer $\left(\mathrm{Mg}^{2+}\right.$ Plus), $4.0 \mu \mathrm{L}$ dNTP Mixture (each

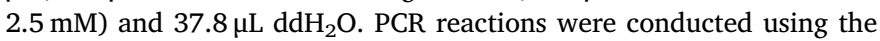
following program: $1 \mathrm{~min}$ for denaturation at $94^{\circ} \mathrm{C}, 30$ cycles of $1 \mathrm{~min}$ at $94^{\circ} \mathrm{C}, 1 \mathrm{~min}$ for annealing at $60^{\circ} \mathrm{C}$, and $1.5 \mathrm{~min}$ for elongation at $72{ }^{\circ} \mathrm{C}$, and a final extension at $72{ }^{\circ} \mathrm{C}$ for $10 \mathrm{~min}$.

PCR conditions for variable regions of int I1 (intI1-V) were: $10 \mathrm{~min}$ for denaturation at $95^{\circ} \mathrm{C}, 35$ cycles of $30 \mathrm{~s}$ at $95^{\circ} \mathrm{C}, 30 \mathrm{~s}$ for annealing at $55{ }^{\circ} \mathrm{C}$, and $5 \mathrm{~min}$ for elongation at $72{ }^{\circ} \mathrm{C}$, and a final extension at $72{ }^{\circ} \mathrm{C}$ for 5 min. PCR products of intI1-V were sent to BGI for sequencing and the sequences were analyzed using NCBI database and integrons database INTEGRALL.

\subsection{Statistical analysis}

Statistical analysis was conducted by SPSS Statistic 19 (IBM, USA) and Origin 2019 (OriginLab, USA). Differences were considered significant when $\mathrm{P}<0.05$. Venn diagram, bar chart, pie chart and heat map were conducted in $\mathrm{R}$ environment. Circos graph was built using Circos-0.67-7. Phylogenetic analysis was conducted by FastTree 2.1.3 with Approximately-Maximum-Likelihood method and constructed with R. ARG cassette assays were visualized by Vector NTI 11.5.1.

\section{Results and discussion}

\subsection{Identification of microplastics}

Among all the collected microplastics, fibers accounted for the maximum proportion (75\%). The abundances of fibers in influent, pond water, and effluent were 53,47 , and 44 items $/ \mathrm{m}^{3}$. The photographs of these fibers exhibited the morphology of the separated fibers (Fig. 1). The FTIR spectra showed that the chemical composition of fibers was polyethylene terephthalate (PET). PET was the common thermoplastic polymer resin of polyester family, which was used for clothing, containers and thermoforming for manufacturing. PET was always used as filter material to make fiber balls in recirculating aquaculture system (RAS) for removing harmful substances and recycling water by filtration, sedimentation, biological oxidation, etc. In this study, fibers were mostly derived from the filter materials in the industrial RAS.

\subsection{Diversity and abundance of cultivable $A R B$}

The numeration results demonstrated that the colony forming units (CFU) of total cultivable bacteria (TCB) were $3.00 \times 10^{6} \mathrm{cfu} / \mathrm{mL}$, $3.10 \times 10^{5} \mathrm{cfu} / \mathrm{mL}$ and $2.90 \times 10^{5} \mathrm{cfu} / \mathrm{mL}$ in the water samples (OW, $\mathrm{PW}$ and IW), and $2.80 \times 10^{8} \mathrm{cfu} / \mathrm{g}, 2.72 \times 10^{8} \mathrm{cfu} / \mathrm{g}$ and $1.44 \times 10^{8} \mathrm{cfu} / \mathrm{g}$ in the microplastic samples (OM, PM and IM). The abundance of cultivable bacteria collected from microplastic samples was 100-5000 times higher than that from water samples. The colony forming units of cultivable ARB were $3.10 \times 10^{5}-2.50 \times 10^{6} \mathrm{cfu} / \mathrm{mL}$, $1.00 \times 10^{4}-2.60 \times 10^{5} \mathrm{cfu} / \mathrm{mL}, \quad 2.00 \times 10^{3}-1.00 \times 10^{5} \mathrm{cfu} / \mathrm{mL} \quad$ in water samples (OW, PW, IW), and $7.68 \times 10^{7}-2.48 \times 10^{8} \mathrm{cfu} / \mathrm{g}$, $4.96 \times 10^{7}-2.08 \times 10^{8} \mathrm{cfu} / \mathrm{g}, 6.40 \times 10^{6}-6.48 \times 10^{7} \mathrm{cfu} / \mathrm{g}$ in microplastic samples (OM, PM, IM) (Fig. 2A). The ratios of ARB to TCB were calculated and listed in Fig. 2B, which showed significant differences in different samples. For the same type of agar plates, the percentage of ARB to TCB in microplastic samples was significantly higher than that in water samples. Among single antibiotic resistant bacteria, the highest percentage of antibiotic resistance was to chloramphenicol (34.5\%-88.6\%), followed by tetracycline $(34.5 \%-85.7 \%)$ and sulfafurazole $(20.7 \%-80.0 \%)$. The percentages of antibiotic resistance to gentamicin $(20.7 \%-71.4 \%)$, ciprofloxacin $(6.9 \%-57.1 \%)$ and penicillin $(10.3 \%-60.0 \%)$ and erythromycin $(6.9 \%-30.7 \%)$ were lower. The lowest percentage of antibiotic resistance was to multiple antibiotics $(0.69 \%-27.43 \%)$. In summary, the percentage of ARB in microplastic samples was obviously higher than that in water samples. In total, $2,416,167$ high quality $16 \mathrm{~S}$ rRNA gene sequences were generated from 54 samples by high-throughput sequencing. After subsampling each sample to an equal sequencing depth and clustering, 2087620 operational taxonomic units (OTUs) at $97 \%$ identity were obtained. Community diversity and richness estimators were showed in Table S3. The community richness estimators (Sobs, Chao1 and ACE) of microplastic samples were higher than those in water samples. The community diversity estimators (Shannon and Simpson) also indicated that the bacteria community diversity of microplastic samples was higher than that 
A
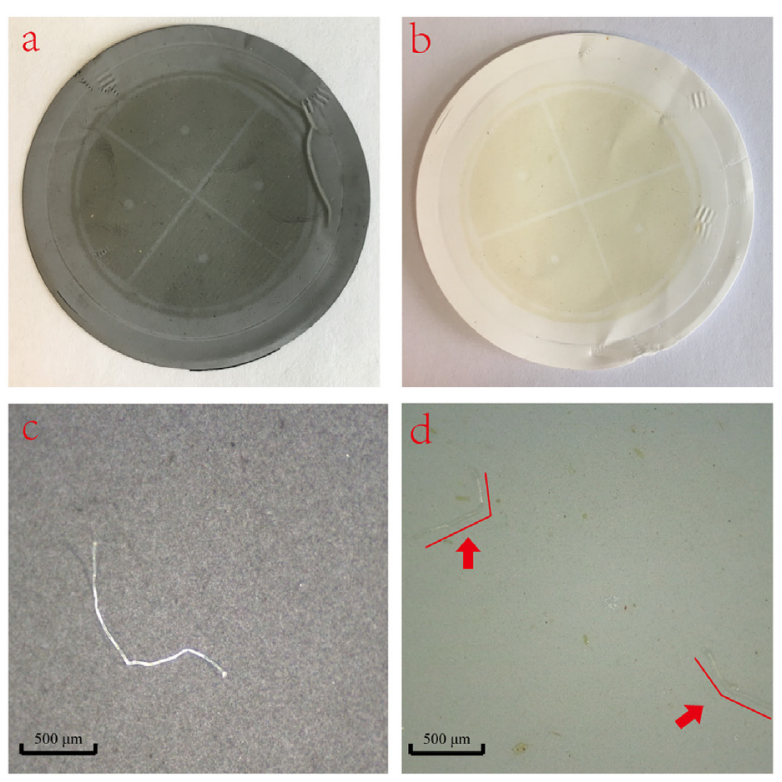

$\mathrm{B}$

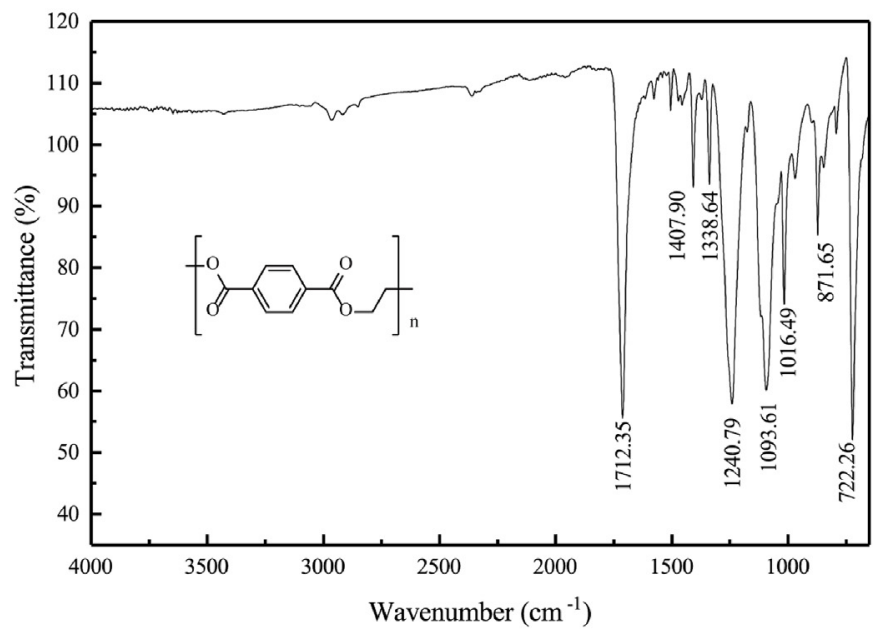

Fig. 1. (A) a \& b, photos of films after filtration of collected microplastics. c \& d, photos of microplastics by stereomicroscope. (B) Fourier Transform Infrared Spectroscopy (FTIR) of the major composition of collected microplastics.

of water samples collected from the same sampling site.

As shown in Fig. S1, the dominant phyla of cultivable bacteria in microplastic samples were similar to those in water samples. On phylum level, Proteobacteria hold the highest abundance (34.8\%-92.2\%), and followed by Bacteroidetes $(6.7 \%-64.5 \%)$. The phyla of Firmicutes $(<3.1 \%)$ and Tenericutes $(<0.7 \%)$ were detected with relatively lower relative abundance. The compositions of bacteria communities on genus level were visualized by circos diagram (Fig. 3A). The predominant genus was Vibrio, followed by Muricauda, Ruegeria and Sunxiuqinia. Other dominant cultivable bacterial genera included Marinobacterium, Marinobacter, Maritalea, Tamlana, Labrenzia, Donghicola and so on. These genera of bacteria were typically found in salt water including oceans, estuaries and saline lakes. The heat map (Fig. 3B) graphically demonstrated that the cultivable bacteria communities and the relative abundance varied from microplastic samples to water samples, which supported the community richness and diversity estimators. Vibrio, Muricauda, Ruegeria, Sunxiuqinia, Marinobacterium, Marinobacter, Tamlana, Labrenzia and Donghicola were dominant genera and their relative abundances in microplastic samples were significantly different from those in water samples. For example, for the same type of antibiotics, the relative abundance of Muricauda,
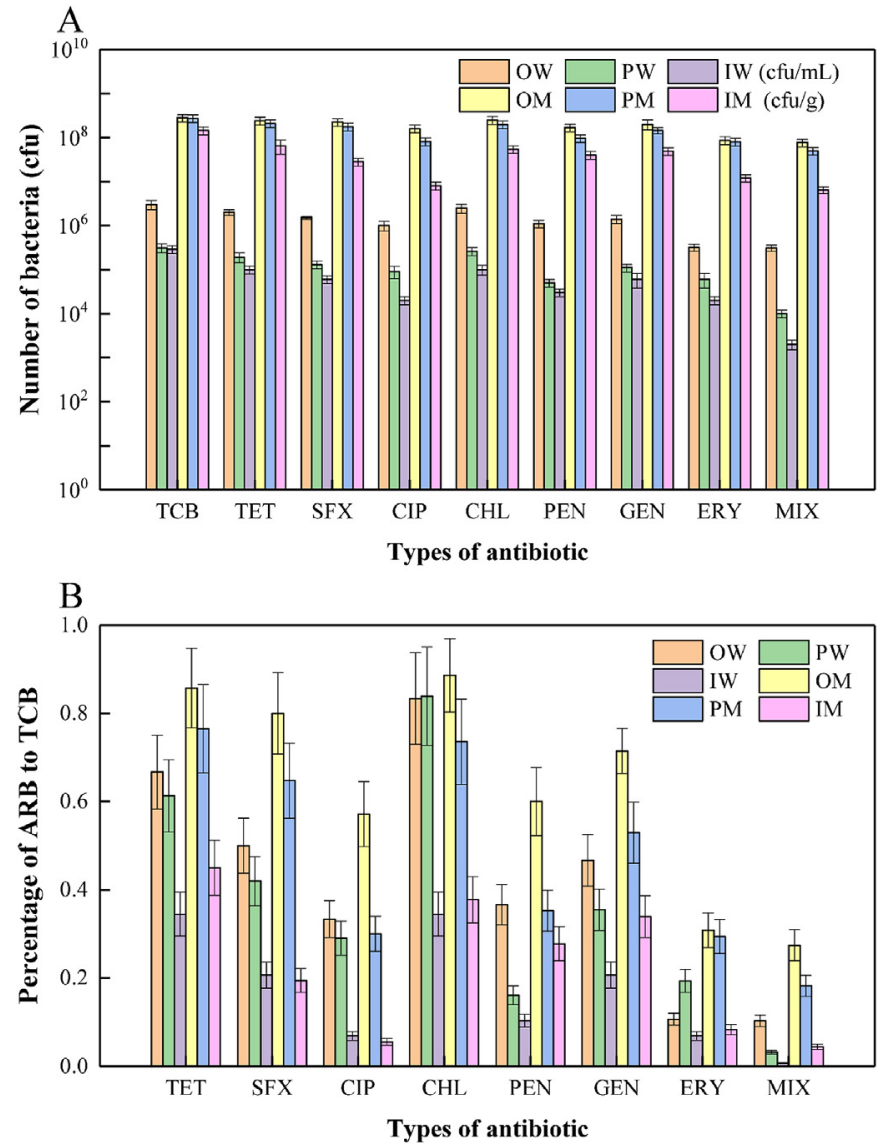

Fig. 2. (A) Numeration of the counts of total cultivable bacteria (TCB) and antibiotic resistant bacteria (ARB) with different types of antibiotics. (B) Percentage of ARB to TCB. TET, tetracycline; SFX, sulfafuxazole; CIP, ciprofloxacin; CHL, chloramphenicol; PEN, penicillin; GEN, gentamicin; ERY, erythromycin; MIX, agar plates with a combination of seven antibiotics. IW, water samples in the inlet; PW, water samples in aquaculture ponds; OW, water samples in the outlet; IM, microplastic samples in the inlet; PM, microplastic samples in aquaculture ponds; OM, microplastic samples in the outlet. CFU/mL, unit of water samples; CFU/g, unit of microplastic samples.

Marinobacter, Ruegeria, Tamlana, Labrenzia and Sunxiuqinia in microplastic samples was higher than that in water samples. A clustering tree of top 30 genera based on the relative abundance was constructed to reveal the relationships between different cultivable ARB. The result revealed that the relative abundance of ARB in microplastic samples was higher than that in water samples. Microplastics could be colonized by different microbial communities and wrapped by biofilms, thus becoming vectors for the selection and spread of bacteria. Pearson's correlations between environmental factors and water samples/microplastic samples (Fig. 4) showed that there were positive and significant correlations between turbidity, silicate, TOC and the counts of cultivable bacteria from water samples, while several positive and significant correlations existed between water quality parameters and the cultivable bacteria of microplastic samples. This result further revealed that the difference between the habitat of microplastic surface and the environment of water phase resulted in the different distribution of ARB in water phase and on the surface of microplastics.

To further investigate the cultivable bacteria collected from agar plates with a combination of seven antibiotics, Venn diagram, pie diagram and phylogenetic tree were established. The Venn diagram (Fig. 5A) showed the differences of cultivable TCB and cultivable ARB from the plates with mixed antibiotics between microplastic samples and water samples. For total cultivable bacterial community, the same 76 OTUs were detected both in water and microplastic samples, and 


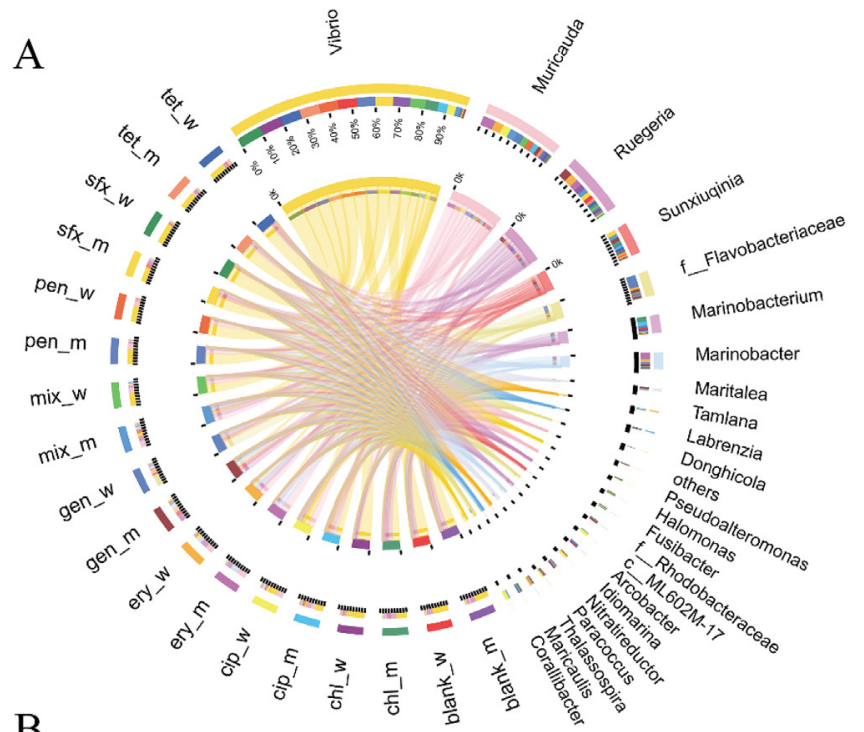

B

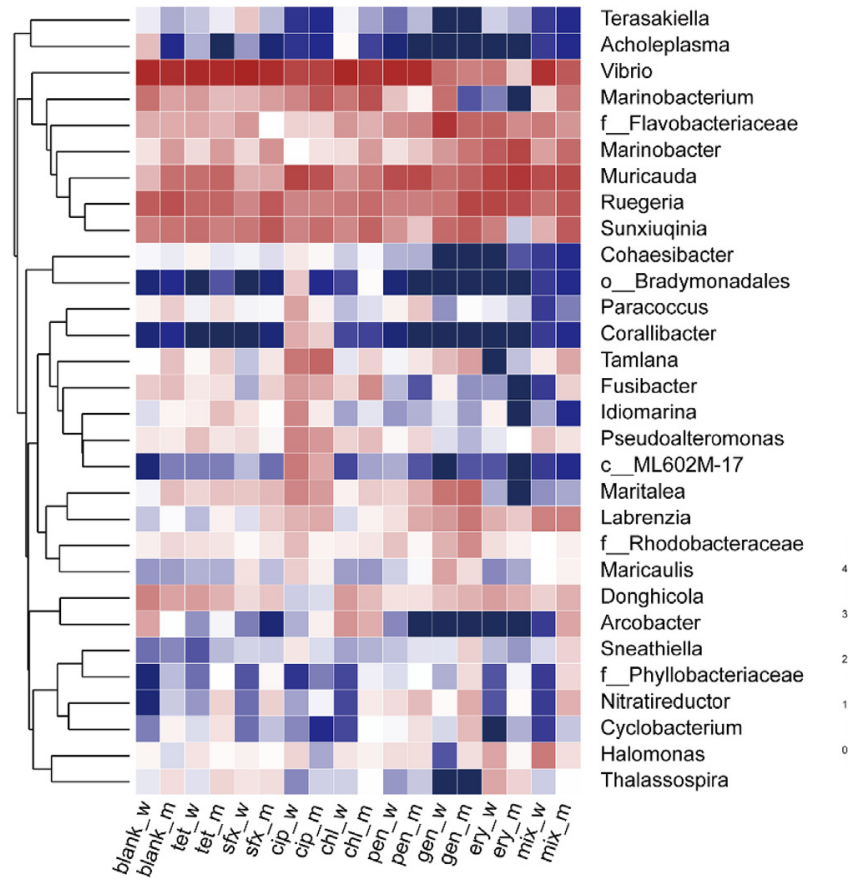

Fig. 3. High-throughput sequencing of microbial community. (A) Circos: distribution and relative abundance of cultivable bacteria collected from different antibiotic resistant plates on genus level. (B) Heatmap: relative abundance of the top 30 bacteria on genus level and the clustering of them. Samples were cultivable bacteria collected from agar plates with different types of antibiotics. Blank_w, plates without antibiotic of water samples; Blank_m, plates without antibiotic of microplastic samples. Mix_w, plates with a combination of seven antibiotics of water samples; Mix_m, plates with a combination of seven antibiotics of microplastic samples.

there were less specific OTUs in water samples (21 OTUs) compared with microplastic samples (42 OTUs). For cultivable bacteria from plates with mixed antibiotics, the same 62 OTUs were detected in water and microplastic samples, and there were less specific OTUs in water samples (11 OTUs) compared with microplastic samples (27 OTUs). Clearly, the abundance and diversity of cultivable bacterial community in microplastic samples was higher than that in water samples. It could be speculated that microplastics might play important roles in bacterial community diversity. The pie diagram (Fig. 5B) showed the bacterial community of cultivable bacteria collected from plates with mixed antibiotics from microplastic samples and water samples. Vibrio,
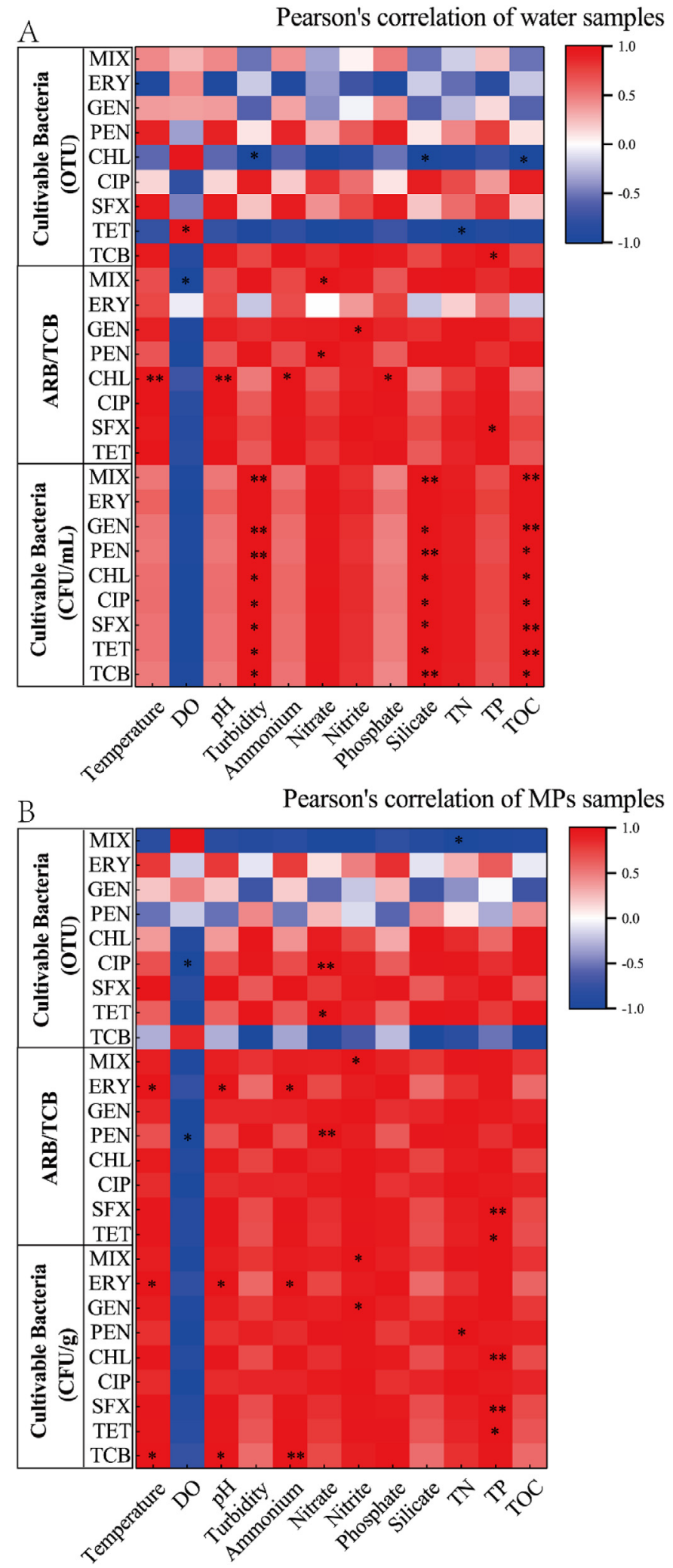

Fig. 4. The Pearson's correlations between environmental factors and water samples/microplastic samples (the counts of cultivable bacteria, the ratios of ARB to TCB, the OTU numbers of cultivable bacteria).

Muricauda, Ruegeria and Sunxiuqinia were dominant cultivable bacteria genera. These bacteria were common bacteria in marine environment and aquaculture system, especially in mariculture system. However, as multi-antibiotic resistant bacteria, the comparisons of their diversity 
A
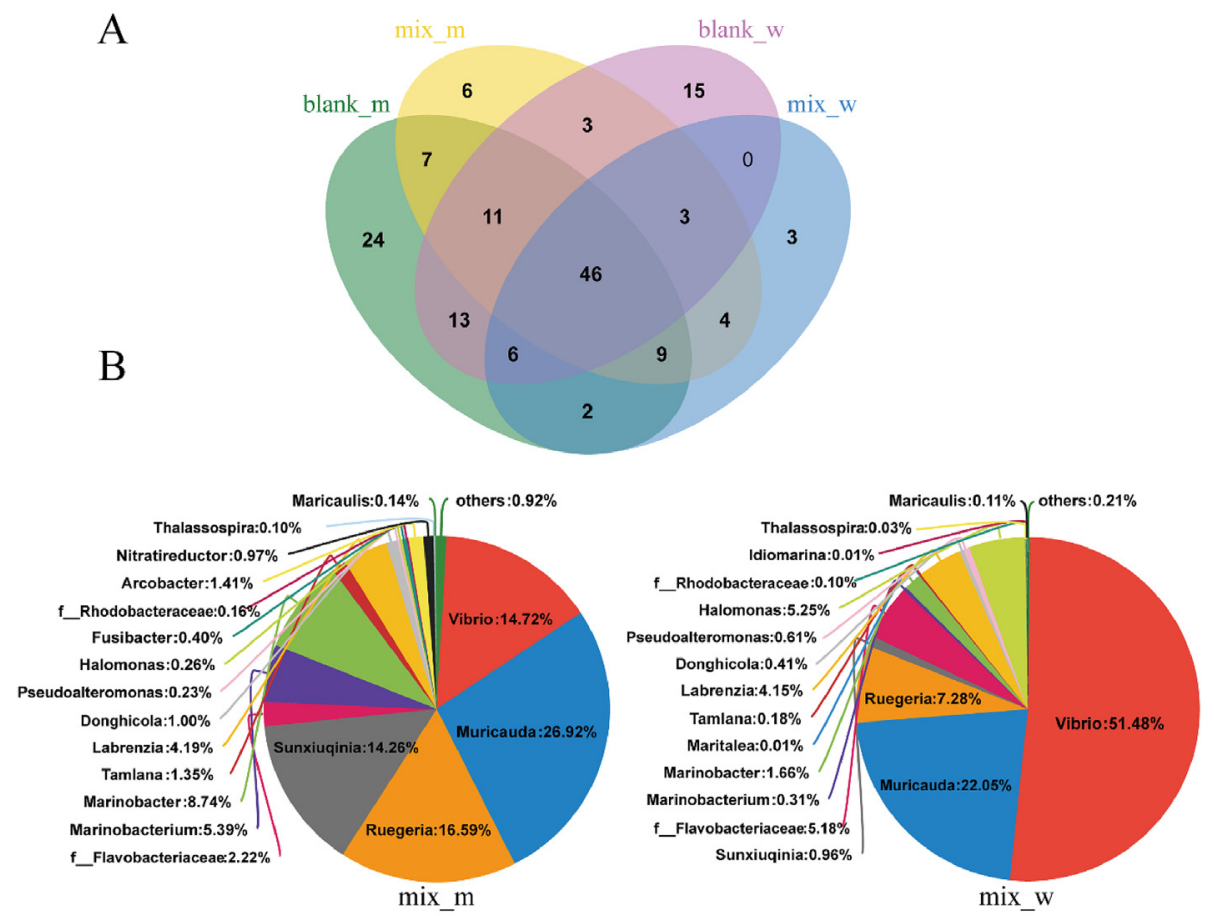

$\mathrm{C}$

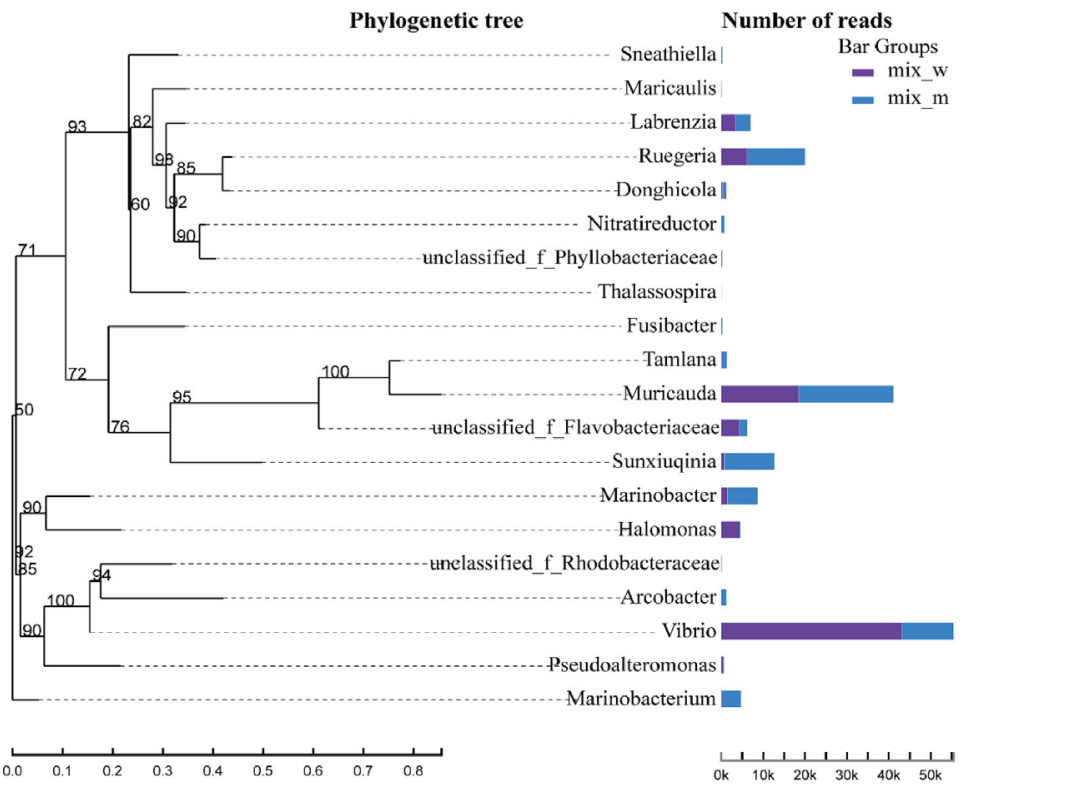

Fig. 5. (A) Venn diagram of cultivable bacteria of blank_w, blank_m, mix_w and mix_m on OTU level. (B) Pie chart of the composition of mix_w and mix_m on genus level. (C) Phylogenetic tree of the relative abundance of top 20 bacteria genera of mix_w and mix_m. Blank_w, plates without antibiotic of water samples; Blank_m, plates without antibiotic of microplastic samples. Mix_w, plates with a combination of seven antibiotics of water samples; Mix_m, plates with a combination of seven antibiotics of microplastic samples.

and abundance in seawater and on the surface of microplastics have not been investigated in previous researches. Vibrio accounted for $51.5 \%$ in water samples (mix_w) and $14.7 \%$ in microplastic samples (mix_m). Vibrio was a genus of gram-negative bacteria. Several species of Vibrio are pathogens and typically found in salt water, which can be carried by numerous marine animals such as crabs or prawns and cause fatal infections in animals and humans during exposure. Microplastics might serve as vectors for dispersal of pathogens such as Vibrio spp. (Zettler et al., 2013). Muricauda accounted for $22.0 \%$ in water sample (mix_w) and $26.9 \%$ in microplastic sample (mix_m). Muricauda, a member of the phylum Bacteroidetes, was isolated from salty lake, coastal hot spring and marine environments including tidal flat sediment, intertidal sediment and crude oil contaminated seawater (Hwang et al., 2009).
Muricauda was important bacteria in aquaculture environment, especially in mariculture areas. The percentage of Ruegeria was $16.6 \%$ in microplastic samples and $7.3 \%$ in water samples. Ruegeria, a genus of phylum Proteobacteria, has been isolated from marine sediments, seawater, surfaces of marine organisms, hypersaline ponds and so on, especially isolated from mariculture systems to pose probiotic potential because of the inhibition of fish pathogens (Sonnenschein et al., 2017). Previous study reported that Sunxiuqinia strains were isolated from shallow sediment such as sea cucumber farm and Nagasuka Fishery Harbor (Qu et al., 2011). This study showed that Sunxiuqinia accounted for $14.3 \%$ in microplastic samples and only $1.0 \%$ in water samples. To further investigate the relationships between the top 20 genera of cultivable ARB in water samples and microplastic samples, a phylogenetic 
tree was constructed according to the representative sequence analysis (Fig. 5C). The reads numbers of each bacteria genus of water samples (mix_w) and microplastic samples (mix_m) were also demonstrated in the phylogenetic tree to suggest that the top 20 bacteria genera from microplastic samples were phylogenetically close to those from water samples. Additionally, the reads number demonstrated the different relative abundance of cultivable ARB between water samples and microplastic samples. Among top 20 genera, the relative abundance of 16 bacteria genera such as Muricauda, Ruegeria, Sunxiuqinia, Marinobacter, Labrenzia, Marinobacterium, etc in microplastic samples was higher than that in water samples. Only four genera (Vibrio, Halomonas, Pseudoalteromonas, etc) were more prevalent in water samples, while they were still detected in microplastic samples. It further confirmed that microplastics influenced the enrichment and evolution of bacterial communities on their surface. Specific bacteria were selectively enriched by microplastic biofilm.

\subsection{MARB isolates in microplastic and water samples}

A total of 210 bacteria isolates were isolated from Zobell 2216E agar plates with a combination of seven antibiotics. Approximately $76.2 \%$ of these isolates were resistant or intermediate to at least three antibiotics, which were recognized as MARB isolates in this investigation. Among all the MARB, isolates from the water/microplastic samples in the influent (IW), mariculture pond (PW) and effluent (OW) accounted for $13.1 \% / 15.6 \%, \quad 14.4 \% / 16.2 \%$ and $18.8 \% / 21.9 \%$, respectively. Compared with the water samples, much more MARB were isolated from the microplastic samples.

The identification of these MARB isolates indicated that the predominant genus was Labrenzia (30.6\%), followed by Vibrio (20.0\%), Ruegeria (12.5\%) and Muricauda (11.3\%). Genera of Tenacibaculum (7.5\%), Tamlana (6.9\%), Maricaulis (3.8\%), Thalassospira (3.1\%), Marinobacter (1.9\%), Alpha-proteobacterium (1.2\%), Roseovarius (0.6\%) and Bacillus $(0.6 \%)$ were also obtained. Similarly, the genera of Labrenzia, Vibrio, Ruegeria, Muricauda, Tamlana and Marinobacter were the main composition of cultivable bacterial communities based on the result of high-throughput sequencing. The genus of Labrenzia accounted for $4.2 \%$ in mixed antibiotic resistant bacterial community while it accounted for $30.6 \%$ among the MARB isolates, which might be caused by the limitations of separation and dilution methods. Species of Labrenzia aggregate accounted for the highest proportion (30.6\%). Labrenzia aggregate is usually isolated from marine habitats, associated with oysters, halophytes, dinoflagellates in previous researches (Camacho et al., 2016). The most prevalent specie was Vibrio alginolyticus in this study, which has been confirmed as important pathogens in mariculture environment. Some dominant bacteria were isolated with higher percentage in the microplastic samples than those in the water sample. For instance, Labrenzia, Vibrio and Tenacibaculum were detected with higher proportion in the microplastic samples (IM) than those in water samples from the influent (IW). Labrenzia, Tenacibaculum, Tamlana, etc, were detected with higher proportion in the microplastic samples (OM) than those in the water samples from the effluent (OW). Ruegeria, Muricauda, Tamlana, etc, were detected with higher percentage in the microplastic samples than those in water samples from the mariculture pond.

\subsection{Antibiotic susceptibility of MARB isolates}

The result of antibiotic susceptibility tests revealed a frequent incidence of cultivable MARB resistant to multiple antibiotics. Among the total MARB isolates, the MARB isolates resistant or intermediate to six antibiotics accounted for $7.5 \%$, isolates resistant or intermediate to five classes of antibiotics accounted for $23.9 \%$, isolates resistant or intermediate to four antibiotics accounted for $52.2 \%$, and $16.4 \%$ isolates were resistant or intermediate to three antibiotics. The proportion of MARB isolates resisting or intermediating to four and three antibiotics were much higher in the microplastic samples than that in the water samples. The highest percentage of antibiotic resistance was to penicillin $(86.8 \%)$, followed by sulfafurazole $(75.8 \%)$, erythromycin $(75.8 \%)$ and tetracycline $(54.9 \%)$. Lower percentage of antibiotic resistance was to chloramphenicol (34.1\%), ciprofloxacin (14.3\%) and gentamicin (6.6\%). Interestingly, the percentage of antibiotic resistance to tetracycline, erythromycin and penicillin was higher in the microplastic samples than that in water samples collected from the influent (IM > IW), the ratio of resistance to sulfafurazole and erythromycin was higher in microplastic samples from the aquaculture pond (PM > PW), and the proportion of resistance to ciprofloxacin, chloramphenicol and gentamicin was higher in microplastic samples from the effluent (OM > OW). It has been reported that microplastics can adsorb antibiotics on their surfaces in aquatic environment, which were well known as the cause to antibiotic resistance (Li et al., 2018). This phenomenon might be partially caused by the different properties of different antibiotics with different concentrations through the process of the industrial mariculture system.

A total of seventeen multiple antibiotic resistance profiles were detected from the MARB isolates, including 6 profiles resistant to three antibiotics, 2 profiles resistant to four antibiotics, 5 profiles resistant to five antibiotics and 3 profiles resistant six antibiotics. The dominant multiple antibiotic resistance profile was TET-SFX-ERY-PEN (49.3\%), which accounted for $25.4 \%$ in microplastic samples and $23.9 \%$ in water samples. The profile of TET-SFX-CHL-ERY-PEN (13.4\%) was also detected with relatively higher percentage. Notably, the profile of TETSFX-ERY-PEN was included in six types of profiles and detected in $73.1 \%$ of the MARB isolates. Considering these common profiles, most of sulfafurazole resistant isolates were also resistant to tetracycline, erythromycin and penicillin. These MARB isolates presented prevalent antibiotic resistance to sulfafurazole, penicillin, tetracycline and erythromycin and they were generally sensitive to ciprofloxacin and gentamicin.

\subsection{Occurrence of ARGs in MARB isolates}

Among the typical MARB isolates, including Muricauda, Ruegeria, Vibrio and Labrenzia (4 isolates from microplastic samples and 4 isolates from water samples), 65 of the 75 ARGs were detected (Fig. 6). These ARGs involved eight classes of antibiotics and four different antibiotic resistant mechanisms: efflux pumps (48/75), cellular protection (8/75), antibiotic deactivation (14/75) and other/unknown (5/75). All the primers have been reported (Zhu et al., 2017). As shown in Fig. 6A and $\mathrm{B}$ and 15 tetracycline resistance genes, 3 sulfonamide resistance genes, 1 quinolone resistance genes, 2 chloramphenicol resistance genes, $4 \beta$ lactamase resistance genes, 5 aminoglycoside resistance genes, 3 macrolide resistance genes and 33 multidrug resistance genes were detected. Nine of these ARGs ( $q n r \mathrm{~B}, q n r \mathrm{~S}, a a c \mathrm{C} 2$, aacC4, acrA, emrB/qacA, $o p r \mathrm{D}, e m r \mathrm{~B} / q a c \mathrm{~A}$ and $s d e \mathrm{~B}$ ) were not detected in these isolates. For MARB isolates from microplastic samples, the positive detection rate of ARGs was up to $80.0 \%(60 / 75)$, while the detection rate of MARB isolates from water samples was $65.3 \%(49 / 75)$.

The average of positive detection rate of $\beta$-lactamase resistance genes was the highest among the MARB isolates, which presented higher positive detection rate in the microplastic samples $(62.5 \%)$ than that in the water samples $(56.3 \%)$. The positive detection ratios of sulfonamide resistance genes (MP 50.0\% > Water 41.7\%) and tetracycline resistance genes (MP $40.0 \%>$ Water $38.3 \%$ ) were second to the $\beta$-lactamase resistance genes. Other types of ARGs were detected with relatively lower proportion: aminoglycoside resistance genes (MP $32.1 \%>$ Water 10.7\%), macrolide resistance genes (MP $33.3 \%>$ Water $8.3 \%$ ), chloramphenicol resistance genes $(12.5 \%)$ and quinolone resistance genes (8.3\%). Multi-antibiotics resistance genes were diverse and prevalent in these MARB isolates, and the positive detection rates of these genes were higher in microplastic samples (34.9\%) than those in water samples (21.7\%). Notably, $84.6 \%$ of all the 
A

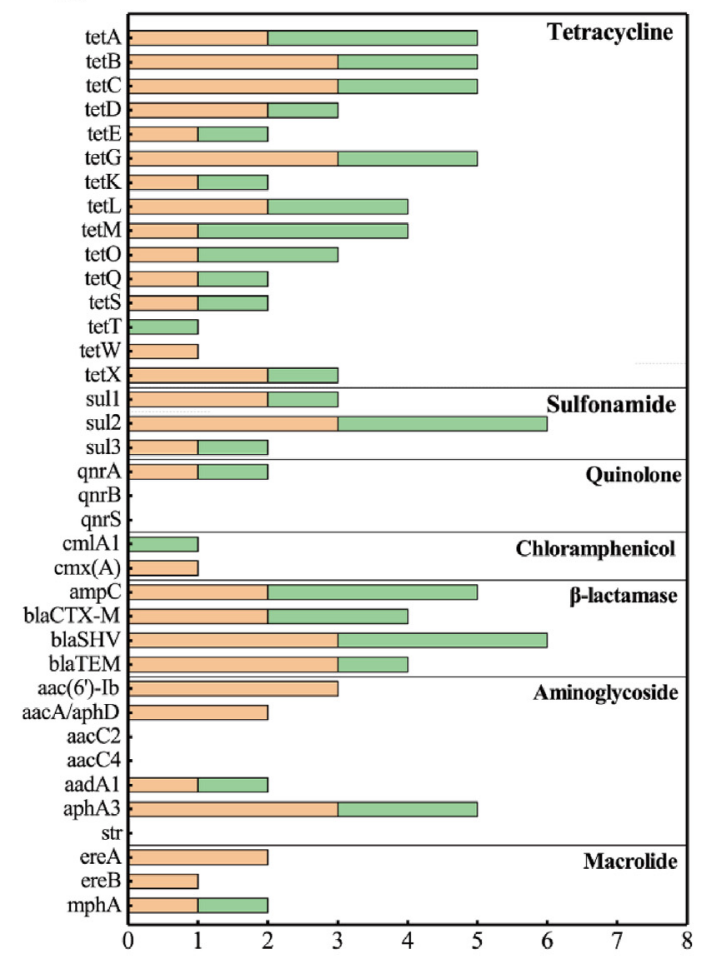

B

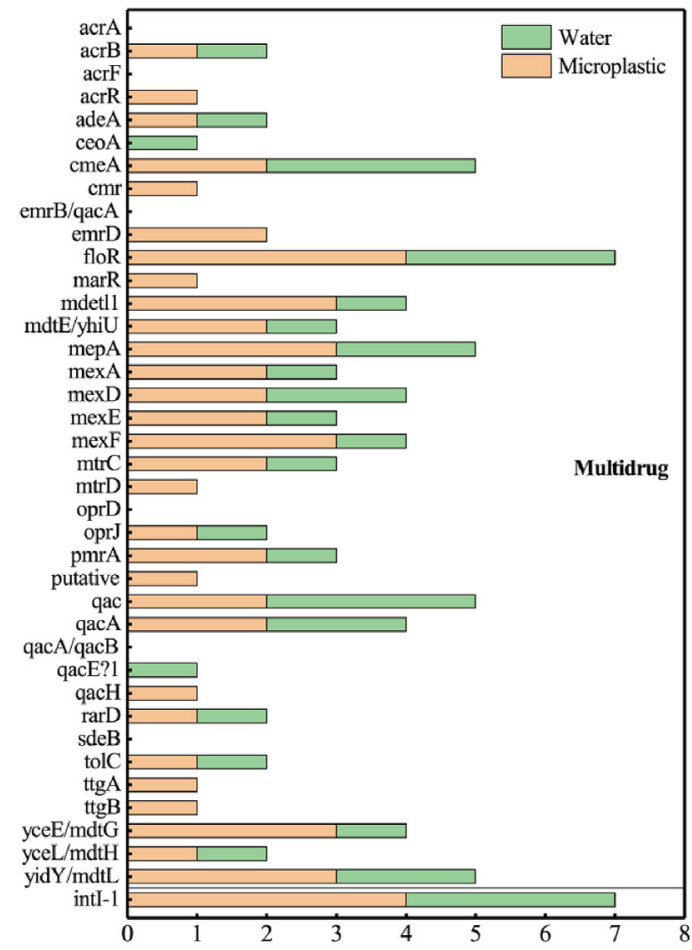

C

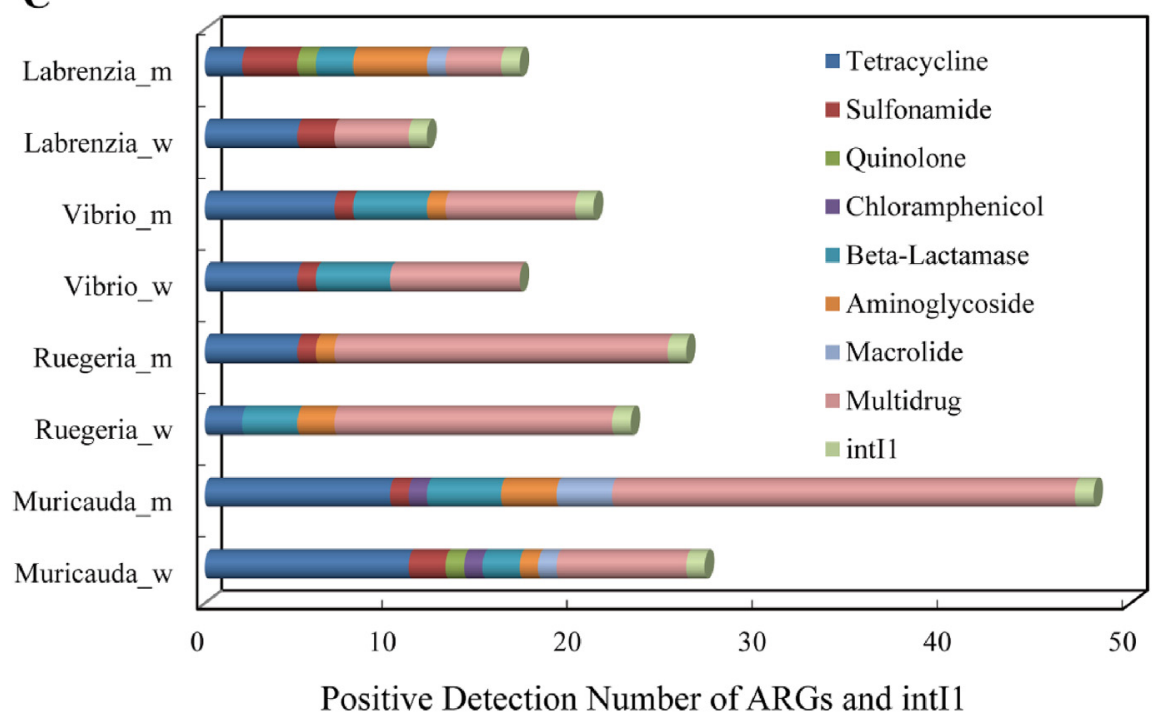

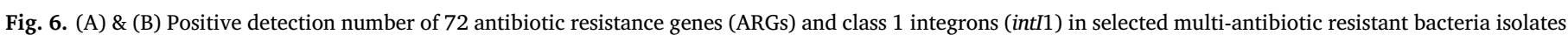

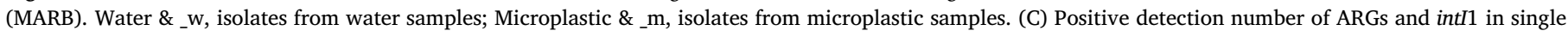
MARB isolates.

target ARGs were detected with higher positive detection rates in the MARB isolates from microplastic samples than those from the water samples. The most frequently detected ARGs were FloR (MP $100.0 \%>$ Water $75.0 \%)$, sul2 $(75.0 \%)$ and blaSHV $(75.0 \%)$. This result revealed that the MARB gathered on the surface of microplastics hold more ARGs as compared to free living bacteria in water environment. Bacteria could form biofilms on the surface of microplastics and serve as the vector for antibiotics and microbial communities, which subsequently led to the proliferation of ARGs on the surface of microplastics.

Considering the ARGs detection results of different bacterial isolates, the distribution of ARGs varied in different bacterial genera and different sources of samples (Fig. 6C). The number of ARGs detected in Muricauda was the highest, followed by Ruegeria and Vibrio. The numbers of ARGs detected in the isolates from MP samples were obviously higher than those from water samples. Multiple antibiotic resistances were demonstrated and multiple ARGs were detected in these MARB isolates. The positive detection result of ARGs was not completely consistent with the antibiotic resistant phenotype from the antibiotic susceptibility tests of these MARB isolates. However, the positive detection rates of $\beta$-lactamases, sulfonamides and tetracyclines resistance genes were higher than others, and the percentage of MARB 
A. Microplastics

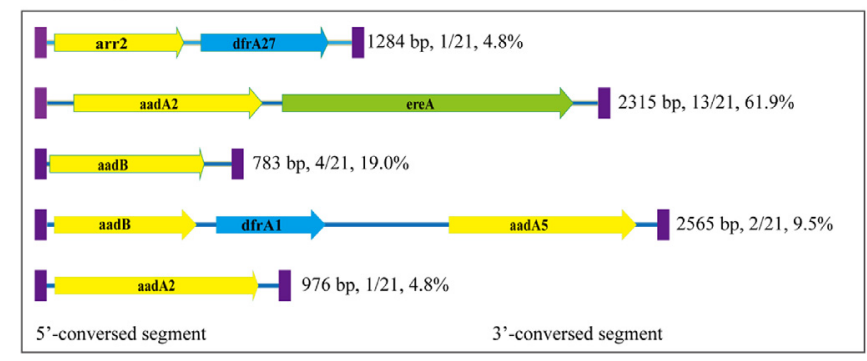

B. Water

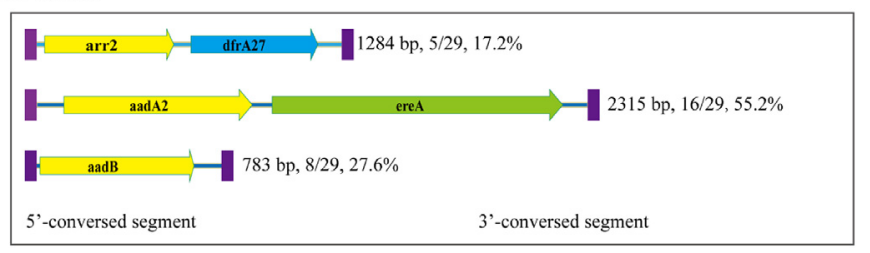

Fig. 7. Class 1 integrons (intI1) associated ARG cassettes in multi-antibiotic resistant bacteria (MARB) isolates. Genes encoding resistance to aminoglycosides streptomycin and spectinomycin (aadA2, aadA5), gentamicin and tobramycin (aadB), trimethoprim (dfrA1 and dfrA27), erythromycin (ereA) and rifampicin (arr2). (A) MARB isolates from microplastic samples. (B) MARB isolates from water samples.

isolates presenting antibiotic resistance to $\beta$-lactamases, sulfonamides and tetracyclines was also higher than that resistant to other types of antibiotics.

\subsection{Occurrence of MGEs of MARB isolates}

Integrons were widely distributed in various species of bacteria and they were bacterial genetic elements that could capture and express genes within mobile gene cassettes. Class 1 integrons (intI1) were almost exclusively responsible for the dissemination of ARGs. In this study, intI 1 was detected in $83.8 \%$ of the 160 MARB isolates. The successfully amplified variable regions of int $I 1$ accounted for $31.2 \%$ in the MARB isolates (50 MARB isolates). A total of five types of intI 1 associated gene cassette arrays were detected, including aadA2 $(2 / 50)$, aadB (12/50), aadA2-ereA (28/50), arr2-dfrA27 (6/50) and aadB-dfrA1aadA5 (2/50) (Fig. 7, Table S4). Seven gene cassettes in the variable region of intI 1 were detected, including those encoding resistance to aminoglycosides streptomycin and spectinomycin (aadA2, aadA5), gentamicin and tobramycin ( $a a d B)$, trimethoprim (dfrA1 and dfrA27), erythromycin (ereA) and rifampicin (arr2), respectively. All the gene cassettes were also reported in previous studies (Malek et al., 2015; Yang et al., 2017).

Interestingly, the numbers of intI 1 associated gene cassette arrays and the types of intI 1 associated gene cassettes detected in the MARB isolates from microplastic samples were higher than those from water samples. Five types of intI 1 associated gene cassette arrays and seven types of intI 1 associated gene cassettes were detected in the microplastic samples, while three types of gene cassette arrays and five types of gene cassettes were detected in the water samples. Given the limitations of experimental methods, it could be speculated that more ARGs and gene cassettes might be carried on these MARB isolates than the detected ones. The prevalence of aadA2 and aadB might be caused by the use of aminoglycosides antibiotics. Gene cassettes of ereA and $d f r A 27$ were prevalent because of the use of macrolide antibiotics and sulfonamide antibiotics, respectively. It was confirmed that the types of intI1 associated gene cassettes were more diverse and abundant from the MARB inhabitants on the surface of microplastics as compared to free-living bacteria in aquatic environment. These results further confirmed that the microplastics acted as the vector for horizontal gene transfer by bacteria. Microplastics were hazardous pollutants which served as hotspot for the enrichment of MARB and vector for the widespread of antibiotic resistance between distinct pathogens.

Based on the diversity and abundance of cultivable ARB, ARGs and MGEs, it could be speculated that pollutants adsorbed on the microplastics might be important factors influencing the fate of ARGs and ARB on the microplastics. Therefore, colonized by diverse and metabolically complex bacterial communities, microplastics are hazardous emerging pollutants which act as hotspots for the evolution of multidrug resistant human pathogen via co-selection and act as potential vector for spreading of bacterial diseases in aquatic environment. It could be speculated that microplastics served as important sinks and much more durable islands with high residence time for ARB and ARGs under complicated conditions in mariculture system, and may enhance the risks to environment and human health. Therefore, action should be taken for the management of microplastic pollution and the abuse of antibiotics, and effective control of microplastics might attribute to the decrease of the abundance of ARB and ARGs.

\section{Conclusion}

The enrichment of ARB, especially MARB, on microplastics in mariculture system was investigated. PET fibers accounted for the highest proportion in the collected microplastics. Both numeration of cultivable ARB and high-throughput sequencing results indicated that the diversity and abundance of cultivable ARB collected from the microplastic samples was higher than that from water samples. The ratio of cultivable ARB to total cultivable bacteria in microplastic samples was higher than that in water samples. A total of 160 MARB isolates were isolated with higher proportion in microplastic samples, and Vibrio, Ruegeria, Muricauda and Labrenzia were important components both in the bacterial communities by sequencing and in the MARB isolates. The higher percentage of antibiotic resistance was to penicillin, sulfafurazole, erythromycin and tetracycline, and multiple antibiotic resistance profile TET-SFX-ERY-PEN accounted for higher proportion in microplastic samples. Furthermore, more ARGs, intI 1 associated gene cassette arrays and gene cassettes were detected in the MARB isolates from microplastic samples compared with free living MARB in water environment. These findings provided initial information on the enrichment of ARB, especially superbugs, on the surface of microplastics, suggesting that microplastics were hazardous pollutants served as the hotspot for MARB and the vector for the spread of antibiotic resistance.

\section{Declaration of competing interest}

The authors declare no competing financial interest.

\section{Acknowledgements}

This work was supported by National Natural Science Foundation of China (No. 41877131), Taishan Scholar Program of Shandong Province (No. tsqn201812116), One Hundred Talents Program of Chinese Academy of Sciences (No. Y629041021), and Two-Hundred Talents Plan of Yantai (No. Y739011021).

\section{Appendix A. Supplementary data}

Supplementary data to this article can be found online at https:// doi.org/10.1016/j.ecoenv.2019.109852.

\section{References}

Browne, M.A., Niven, S.J., Galloway, T.S., Rowland, S.J., Thompson, R.C., 2013.

Microplastic moves pollutants and additives to worms, reducing functions linked to health and biodiversity. Curr. Biol. 23, 2388-2392.

Camacho, M., Redondo-Gómez, S., Rodríguez-Llorente, I., Rohde, M., Spröer, C., 
Schumann, P., et al., 2016. Labrenzia salina sp. nov., isolated from the rhizosphere of the halophyte Arthrocnemum macrostachyum. Int. J. Syst. Evol. Microbiol. 66, 5173-5180.

Frias, J.P.G.L., Sobral, P., Ferreira, A.M., 2010. Organic pollutants in microplastics from two beaches of the Portuguese coast. Mar. Pollut. Bull. 60, 1988-1992.

Harrison, J.P., Schratzberger, M., Sapp, M., Osborn, A.M., 2014. Rapid bacterial colonization of low-density polyethylene microplastics in coastal sediment microcosms. BMC Microbiol. 14, 232.

Hidalgo, R.V., Gutow, L., Thompson, R.C., Thiel, M., 2012. Microplastics in the marine environment: a review of the methods used for identification and quantification. Environ. Sci. Technol. 46, 3060-3075.

Hirai, H., Takada, H., Ogata, Y., Yamashita, R., Mizukawa, K., Saha, M., et al., 2011. Organic micropollutants in marine plastics debris from the open ocean and remote and urban beaches. Mar. Pollut. Bull. 62, 1683-1692.

Hwang, C.Y., Kim, M.H., Bae, G.D., Zhang, G.I., Kim, Y.H., Cho, B.C., 2009. Muricauda olearia sp. nov., isolated from crude-oil-contaminated seawater, and emended description of the genus Muricauda. Int. J. Syst. Evol. Microbiol. 59, 1856-1861.

Kiessling, T., Gutow, L., Thiel, M., 2015. Marine litter as habitat and dispersal vector. In: Bergmann, M., Gutow, L., Klages, M. (Eds.), Marine Anthropogenic Litter. Springer International Publishing, Cham, pp. 141-181.

Law, K.L., Thompson, R.C., 2014. Microplastics in the seas. Science 345, 144-145.

Lechner, A., Keckeis, H., Lumesberger Loisl, F., Zens, B., Krusch, R., Tritthart, M., et al., 2014. The Danube so colourful: a potpourri of plastic litter outnumbers fish larvae in Europe's second largest river. Environ. Pollut. 188, 177-181.

Li, J., Zhang, K., Zhang, H., 2018. Adsorption of antibiotics on microplastics. Environ. Pollut. 237, 460-467.

Lobelle, D., Cunliffe, M., 2011. Early microbial biofilm formation on marine plastic debris. Mar. Pollut. Bull. 62, 197-200.

Lu, J., Wu, J., Zhang, C., Zhang, Y., Lin, Y., Luo, Y., 2018. Occurrence, distribution, and ecological-health risks of selected antibiotics in coastal waters along the coastline of China. Sci. Total Environ. 644, 1469-1476.

Lu, J., Zhang, Y., Wu, J., Wang, J., Zhang, C., Lin, Y., 2019. Occurrence and spatial distribution of antibiotic resistance genes in the Bohai Sea and Yellow Sea areas, China. Environ. Pollut. 252, 450-460.

Lusher, A.L., Tirelli, V., O'Connor, I., Officer, R., 2015. Microplastics in Arctic polar waters: the first reported values of particles in surface and sub-surface samples. Sci. Rep. 5, 14947.

Malek, M.M., Amer, F.A., Allam, A.A., El Sokkary, R.H., Gheith T, Arafa M.A., 2015. Occurrence of classes I and II integrons in enterobacteriaceae collected from zagazig university hospitals, Egypt. Front. Microbiol. 6, 601.

Pruden, A., Pei, R., Heather Storteboom, A., Carlson, K.H., 2006. Antibiotic resistance genes as emerging Contaminants: studies in northern Colorado $\uparrow$. Environ. Sci.
Technol. 40, 7445 .

Qu, L., Zhu, F., Hong, X., Gao, W., Chen, J., Sun, X., 2011. Sunxiuqinia elliptica gen. nov., sp. nov., a member of the phylum Bacteroidetes isolated from sediment in a sea cucumber farm. Int. J. Syst. Evol. Microbiol. 61, 2885-2889.

Reisser, J., Shaw, J., Hallegraeff, G., Proietti, M., Barnes, D.K., Thums, M., et al., 2014. Millimeter-sized marine plastics: a new pelagic habitat for microorganisms and invertebrates. PLoS One 9, e100289.

Rochman, C.M., 2013. Plastics and priority pollutants: a multiple stressor in aquatic habitats. Environ. Sci. Technol. 47, 2439-2440.

Rochman, C.M., 2015. The complex mixture, fate and toxicity of chemicals associated with plastic debris in the marine environment. In: Bergmann, M., Gutow, L., Klages, M. (Eds.), Marine Anthropogenic Litter. Springer International Publishing, Cham, pp. 117-140.

Sonnenschein, E.C., Nielsen, K.F., D'Alvise, P., Porsby, C.H., Melchiorsen, J., Heilmann, J., et al., 2017. Global occurrence and heterogeneity of the Roseobacter-clade species Ruegeria mobilis. Int. Soc. Microb. Ecol. J. 11, 569-583.

Syberg, K., Khan, F.R., Selck, H., Palmqvist, A., Banta, G.T., Daley, J., et al., 2015. Microplastics: addressing ecological risk through lessons learned. Environ. Toxicol. Chem. 34, 945-953.

Thompson, R.C., Olsen, Y., Mitchell, R.P., Davis, A., Rowland, S.J., John, A.W.G., et al., 2004. Lost at sea: where is all the plastic? Science 304 838-838.

Wang, J.H., Lu, J., Wu, J., Zhang, Y.X., Zhang, C., 2019. Proliferation of antibiotic resistance genes in coastal recirculating mariculture system. Environ. Pollut. 248, $462-470$.

Wang, J.H., Lu, J., Zhang, Y.X., Wu, J., Luo, Y., Liu, H., 2018. Metagenomic analysis of antibiotic resistance genes in coastal industrial mariculture systems. Bioresour. Technol. 253, 235-243.

Wayne, P.A., 2011. Clinical and Laboratory Standards Institute. Performance Standards for Antimicrobial Susceptibility Testing.

Wright, S.L., Thompson, R.C., Galloway, T.S., 2013. The physical impacts of microplastics on marine organisms: a review. Environ. Pollut. 178, 483-492.

Yang, Q., Tian, T., Niu, T., Wang, P., 2017. Molecular characterization of antibiotic resistance in cultivable multidrug-resistant bacteria from livestock manure. Environ. Pollut. 229, 188-198.

Zettler, E.R., Mincer, T.J., Amaral-Zettler, L.A., 2013. Life in the "plastisphere": microbial communities on plastic marine debris. Environ. Sci. Technol. 47, 7137-7146.

Zhu, Y.G., Zhao, Y., Li, B., Huang, C.L., Zhang, S.Y., Yu, S., et al., 2017. Continental-scale pollution of estuaries with antibiotic resistance genes. Nat. Microbiol. 2, 16270.

Zhu, Y.G., Johnson, T.A., Su, J.Q., Qiao, M., Guo, G.X., Stedtfeld, R.D., et al., 2013. Diverse and abundant antibiotic resistance genes in Chinese swine farms. In: Proceedings of the National Academy of Sciences of the United States of America. vol. 110. pp. 3435-3440. 